\title{
OrthoBoard: An Orthopaedic App for Medical Education
}

Goh Kian Liang ${ }^{1}$, Mohd Shahidan Noor Rahin ${ }^{1}$, Mohd Shukrimi Awang ${ }^{1}$, Ardilla Hanim Abdul Razak

${ }^{1}$ Department of Orthopaedics, Traumatology and Rehabilitation, Kulliyyah of Medicine, International Islamic University Malaysia

Presenter: Goh Kian Liang

Introduction: Use of mobile apps as a pedagogical tool in the medical education has gained popularity as mobile apps have great potentials in perpetuating clinical knowledge and skills. To leverage mobile technology in medical education, we designed a hybrid mobile app based on our undergraduate orthopaedic curriculum. Materials and Methods: The frontend was created using hybrid mobile framework to target both iOS and Android platforms. The backend is powered by Firebase (Google Inc.) to manage authentication and social messaging. The content consisted of basic orthopaedic skills, trauma and resuscitation skills, Islamic input in orthopaedic and information cheatsheets. Results: OrthoBoard is being used by IIUM medical undergraduates as an ancillary source of learning, particularly useful as quick references and visual guides for enhancing orthopaedic knowledge and performing clinical procedures. The social messaging capability built into the app serves as a platform for discussion and exchange of information. This is the first medical app, to our knowledge, that integrates orthopaedic clinical knowledge and practice as well as Islamic input in orthopaedics into teaching and learning with the integration of social messaging as a platform for enhancement of knowledge and practice. Conclusion: Use of mobile apps as pedagogical tool in the medical education will undoubtedly increase in the future. As students and teachers embrace mobile technology in and outside the classroom, better user-centric and subject-specific apps will be required to fulfil the needs. For medical educators, developing mobile apps will possibly be one of the future armamentarium in the delivery of knowledge and skills. 